\title{
The Optimization on Ranks and Inertias of a Quadratic Hermitian Matrix Function and Its Applications
}

\author{
Yirong Yao \\ Department of Mathematics, Shanghai University, Shanghai 200444, China \\ Correspondence should be addressed to Yirong Yao; yryao@staff.shu.edu.cn
}

Received 3 December 2012; Accepted 9 January 2013

Academic Editor: Yang Zhang

Copyright (C) 2013 Yirong Yao. This is an open access article distributed under the Creative Commons Attribution License, which permits unrestricted use, distribution, and reproduction in any medium, provided the original work is properly cited.

\begin{abstract}
We solve optimization problems on the ranks and inertias of the quadratic Hermitian matrix function $Q-X P X^{*}$ subject to a consistent system of matrix equations $A X=C$ and $X B=D$. As applications, we derive necessary and sufficient conditions for the solvability to the systems of matrix equations and matrix inequalities $A X=C, X B=D$, and $X P X^{*}=(>,<, \geq$, $\leq) Q$ in the Löwner partial ordering to be feasible, respectively. The findings of this paper widely extend the known results in the literature.
\end{abstract}

\section{Introduction}

Throughout this paper, we denote the complex number field by $\mathbb{C}$. The notations $\mathbb{C}^{m \times n}$ and $\mathbb{C}_{h}^{m \times m}$ stand for the sets of all $m \times n$ complex matrices and all $m \times m$ complex Hermitian matrices, respectively. The identity matrix with an appropriate size is denoted by $I$. For a complex matrix $A$, the symbols $A^{*}$ and $r(A)$ stand for the conjugate transpose and the rank of $A$, respectively. The Moore-Penrose inverse of $A \in \mathbb{C}^{m \times n}$, denoted by $A^{\dagger}$, is defined to be the unique solution $X$ to the following four matrix equations

$$
\begin{array}{cl}
\text { (1) } A X A=A, & \text { (2) } X A X=X, \\
\text { (3) }(A X)^{*}=A X, & \text { (4) }(X A)^{*}=X A .
\end{array}
$$

Furthermore, $L_{A}$ and $R_{A}$ stand for the two projectors $L_{A}=$ $I-A^{\dagger} A$ and $R_{A}=I-A A^{\dagger}$ induced by $A$, respectively. It is known that $L_{A}=L_{A}^{*}$ and $R_{A}=R_{A}^{*}$. For $A \in \mathbb{C}_{h}^{m \times m}$, its inertia

$$
\mathbb{\square}_{n}(A)=\left(i_{+}(A), i_{-}(A), i_{0}(A)\right)
$$

is the triple consisting of the numbers of the positive, negative, and zero eigenvalues of $A$, counted with multiplicities, respectively. It is easy to see that $i_{+}(A)+i_{-}(A)=r(A)$. For two Hermitian matrices $A$ and $B$ of the same sizes, we say $A>B(A \geq B)$ in the Löwner partial ordering if $A-B$ is positive (nonnegative) definite.
The investigation on maximal and minimal ranks and inertias of linear and quadratic matrix function is active in recent years (see, e.g., [1-24]). Tian [21] considered the maximal and minimal ranks and inertias of the Hermitian quadratic matrix function

$$
h(X)=A X B X^{*} A^{*}+A X C+C^{*} X^{*} A^{*}+D,
$$

where $B$ and $D$ are Hermitian matrices. Moreover, Tian [22] investigated the maximal and minimal ranks and inertias of the quadratic Hermitian matrix function

$$
f(X)=Q-X P X^{*}
$$

such that $A X=C$.

The goal of this paper is to give the maximal and minimal ranks and inertias of the matrix function (4) subject to the consistent system of matrix equations

$$
A X=C, \quad X B=D,
$$

where $Q \in \mathbb{C}_{h}^{n \times n}, P \in \mathbb{C}_{h}^{p \times p}$ are given complex matrices. As applications, we consider the necessary and sufficient 
conditions for the solvability to the systems of matrix equations and inequality

$$
\begin{array}{lll}
A X=C, & X B=D, & X P X^{*}=Q, \\
A X=C, & X B=D, & X P X^{*}>Q, \\
A X=C, & X B=D, & X P X^{*}<Q, \\
A X=C, & X B=D, & X P X^{*} \geq Q, \\
A X=C, & X B=D, & X P X^{*} \leq Q,
\end{array}
$$

in the Löwner partial ordering to be feasible, respectively.

\section{The Optimization on Ranks and Inertias of (4) Subject to (5)}

In this section, we consider the maximal and minimal ranks and inertias of the quadratic Hermitian matrix function (4) subject to (5). We begin with the following lemmas.

Lemma 1 (see [3]). Let $A \in \mathbb{C}_{h}^{m \times m}, B \in \mathbb{C}^{m \times p}$, and $C \in \mathbb{C}^{q \times m}$ be given and denote

$$
\begin{array}{ccc}
P_{1}=\left[\begin{array}{cc}
A & B \\
B^{*} & 0
\end{array}\right], & P_{2}=\left[\begin{array}{cc}
A & C^{*} \\
C & 0
\end{array}\right], \\
P_{3}=\left[\begin{array}{ccc}
A & B & C^{*} \\
B^{*} & 0 & 0
\end{array}\right], & P_{4}=\left[\begin{array}{ccc}
A & B & C^{*} \\
C & 0 & 0
\end{array}\right] .
\end{array}
$$

Then

$$
\begin{aligned}
& \max _{Y \in \mathbb{C}^{P \times q}} r\left[A-B Y C-(B Y C)^{*}\right] \\
& =\min \left\{r\left[\begin{array}{lll}
A & B & C^{*}
\end{array}\right], r\left(P_{1}\right), r\left(P_{2}\right)\right\}, \\
& \min _{Y \in \mathbb{C}^{p \times q}} r\left[A-B Y C-(B Y C)^{*}\right] \\
& =2 r\left[\begin{array}{lll}
A & B & C^{*}
\end{array}\right] \\
& +\max \left\{w_{+}+w_{-}, g_{+}+g_{-}, w_{+}+g_{-}, w_{-}+g_{+}\right\}, \\
& \max _{Y \in \mathbb{C}^{p \times q}} i_{ \pm}\left[A-B Y C-(B Y C)^{*}\right]=\min \left\{i_{ \pm}\left(P_{1}\right), i_{ \pm}\left(P_{2}\right)\right\} \text {, } \\
& \min _{Y \in \mathbb{C}^{p \times q}} i_{ \pm}\left[A-B Y C-(B Y C)^{*}\right] \\
& =r\left[\begin{array}{lll}
A & B & C^{*}
\end{array}\right]+\max \left\{i_{ \pm}\left(P_{1}\right)-r\left(P_{3}\right), i_{ \pm}\left(P_{2}\right)-r\left(P_{4}\right)\right\},
\end{aligned}
$$

where

$$
w_{ \pm}=i_{ \pm}\left(P_{1}\right)-r\left(P_{3}\right), \quad g_{ \pm}=i_{ \pm}\left(P_{2}\right)-r\left(P_{4}\right) .
$$

Lemma 2 (see [4]). Let $A \in \mathbb{C}^{m \times n}, B \in \mathbb{C}^{m \times k}, C \in \mathbb{C}^{l \times n}, D \in$ $\mathbb{C}^{m \times p}, E \in \mathbb{C}^{q \times n}, Q \in \mathbb{C}^{m_{1} \times k}$, and $P \in \mathbb{C}^{l \times n_{1}}$ be given. Then

(1) $r(A)+r\left(R_{A} B\right)=r(B)+r\left(R_{B} A\right)=r\left[\begin{array}{ll}A & B\end{array}\right]$,

(2) $r(A)+r\left(C L_{A}\right)=r(C)+r\left(A L_{C}\right)=r\left[\begin{array}{l}A \\ C\end{array}\right]$,

(3) $r(B)+r(C)+r\left(R_{B} A L_{C}\right)=r\left[\begin{array}{ll}A & B \\ C & 0\end{array}\right]$,

(4) $r(P)+r(Q)+r\left[\begin{array}{cc}A & B L_{Q} \\ R_{P} C & 0\end{array}\right]=r\left[\begin{array}{lll}A & B & 0 \\ C & 0 & P \\ 0 & Q & 0\end{array}\right]$,

(5) $r\left[\begin{array}{cc}R_{B} A L_{C} & R_{B} D \\ E L_{C} & 0\end{array}\right]+r(B)+r(C)=r\left[\begin{array}{lll}A & D & B \\ E & 0 & 0 \\ C & 0 & 0\end{array}\right]$.

Lemma 3 (see [23]). Let $A \in \mathbb{C}_{h}^{m \times m}, B \in \mathbb{C}^{m \times n}, C \in \mathbb{C}_{h}^{n \times n}$, $Q \in \mathbb{C}^{m \times n}$, and $P \in \mathbb{C}^{p \times n}$ be given, and, $T \in \mathbb{C}^{m \times m}$ be nonsingular. Then

(1) $i_{ \pm}\left(T A T^{*}\right)=i_{ \pm}(A)$,

(2) $i_{ \pm}\left[\begin{array}{cc}A & 0 \\ 0 & C\end{array}\right]=i_{ \pm}(A)+i_{ \pm}(C)$,

(3) $i_{ \pm}\left[\begin{array}{cc}0 & Q \\ Q^{*} & 0\end{array}\right]=r(Q)$,

(4) $i_{ \pm}\left[\begin{array}{cc}A & B L_{P} \\ L_{P} B^{*} & 0\end{array}\right]+r(P)=i_{ \pm}\left[\begin{array}{ccc}A & B & 0 \\ B^{*} & 0 & P^{*} \\ 0 & P & 0\end{array}\right]$.

Lemma 4. Let $A, C, B$, and $D$ be given. Then the following statements are equivalent.

(1) System (5) is consistent.

(2) Let

$$
r\left[\begin{array}{ll}
A & C
\end{array}\right]=r(A), \quad\left[\begin{array}{l}
D \\
B
\end{array}\right]=r(B), \quad A D=C B
$$

In this case, the general solution can be written as

$$
X=A^{\dagger} C+L_{A} D B^{\dagger}+L_{A} V R_{B},
$$

where $V$ is an arbitrary matrix over $\mathbb{C}$ with appropriate size.

Now we give the fundamental theorem of this paper. 
Theorem 5. Let $f(X)$ be as given in (4) and assume that $A X=$ $C$ and $X B=D$ in (5) is consistent. Then

$$
\begin{aligned}
& \max _{A X=C, X B=D} r\left(Q-X P X^{*}\right) \\
& =\min \left\{n+r\left[\begin{array}{ccc}
0 & P & P \\
A Q & C P & 0 \\
-D^{*} & 0 & B^{*}
\end{array}\right]-r(A)-r(B)\right. \\
& -r(P), 2 n+r\left(A Q A^{*}-C P C^{*}\right) \\
& \left.-2 r(A), r\left[\begin{array}{ccc}
Q & 0 & D \\
0 & -P & B \\
D^{*} & B^{*} & 0
\end{array}\right]-2 r(B)\right\}, \\
& \min _{A X=C, X B=D} r\left(Q-X P X^{*}\right) \\
& =2 n+2 r\left[\begin{array}{ccc}
0 & P & P \\
A Q & C P & 0 \\
-D^{*} & 0 & B^{*}
\end{array}\right]-2 r(A)-2 r(B)-r(P) \\
& +\max \left\{s_{+}+s_{-}, t_{+}+t_{-}, s_{+}+t_{-}, s_{-}+t_{+}\right\} \text {, } \\
& \max _{A X=C, X B=D^{ \pm}} i_{ \pm}\left(Q-X P X^{*}\right) \\
& =\min \left\{n+i_{ \pm}\left(A Q A^{*}-C P C^{*}\right)\right. \\
& \left.-r(A), i_{ \pm}\left[\begin{array}{ccc}
Q & 0 & D \\
0 & -P & B \\
D^{*} & B^{*} & 0
\end{array}\right]-r(B)\right\} \\
& \min _{A X=C, X B=D} i_{ \pm}\left(Q-X P X^{*}\right) \\
& =n+r\left[\begin{array}{ccc}
0 & P & P \\
A Q & C P & 0 \\
-D^{*} & 0 & B^{*}
\end{array}\right]-r(A)-r(B) \\
& -i_{ \pm}(P)+\max \left\{s_{ \pm}, t_{ \pm}\right\} \text {, }
\end{aligned}
$$

where

$$
\begin{gathered}
s_{ \pm}=-n+r(A)-i_{\mp}(P)+i_{ \pm}\left(A Q A^{*}-C P C^{*}\right)-r\left[\begin{array}{cc}
C P & A Q A^{*} \\
B^{*} & D^{*} A^{*}
\end{array}\right], \\
t_{ \pm}=-n+r(A)-i_{\mp}(P)+i_{ \pm}\left[\begin{array}{ccc}
Q & 0 & D \\
0 & -P & B \\
D^{*} & B^{*} & 0
\end{array}\right]-\left[\begin{array}{ccc}
0 & P & B \\
A Q & C P & 0 \\
D^{*} & B^{*} & 0
\end{array}\right] .
\end{gathered}
$$

Proof. It follows from Lemma 4 that the general solution of (4) can be expressed as

$$
X=X_{0}+L_{A} V R_{B}
$$

where $V$ is an arbitrary matrix over $\mathbb{C}$ and $X_{0}$ is a special solution of (5). Then

$$
Q-X P X^{*}=Q-\left(X_{0}+L_{A} V R_{B}\right) P\left(X_{0}+L_{A} V R_{B}\right)^{*} .
$$

Note that

$$
\begin{gathered}
r\left[Q-\left(X_{0}+L_{A} V R_{B}\right) P\left(X_{0}+L_{A} V R_{B}\right)^{*}\right] \\
=r\left[\begin{array}{c}
Q \\
\left.P\left(X_{0}+L_{A} V R_{B}\right)^{*}+L_{A} V R_{B}\right) P
\end{array}\right]-r(P) \\
=r\left[\begin{array}{cc}
Q & X_{0} P \\
P X_{0}^{*} & P
\end{array}\right]+\left[\begin{array}{c}
L_{A} \\
0
\end{array}\right] V\left[\begin{array}{ll}
0 & R_{B} P
\end{array}\right] \\
+\left(\left[\begin{array}{c}
L_{A} \\
0
\end{array}\right] V\left[\begin{array}{ll}
0 & R_{B} P
\end{array}\right)^{*}\right]-r(P), \\
i_{ \pm}\left[Q-\left(X_{0}+L_{A} V R_{B}\right) P\left(X_{0}+L_{A} V R_{B}\right)^{*}\right] \\
=i_{ \pm}\left[\begin{array}{c}
Q \\
P\left(X_{0}+L_{A} V R_{B}\right)^{*}
\end{array}\right]-i_{ \pm}(P) \\
=i_{ \pm}\left[\begin{array}{cc}
Q & X_{0} P \\
P X_{0}^{*} & P
\end{array}\right]+\left[\begin{array}{cc}
L_{A} \\
0
\end{array}\right] V\left[\begin{array}{ll}
0 & R_{B} P
\end{array}\right] \\
\left.+\left(\left[\begin{array}{cc}
L_{A} \\
0
\end{array}\right] V\left[\begin{array}{ll}
0 & R_{B} P
\end{array}\right]\right)^{*}\right]-i_{ \pm}(P) .
\end{gathered}
$$

Let

$$
\begin{aligned}
q(V)= & {\left[\begin{array}{cc}
Q & X_{0} P \\
P X_{0}^{*} & P
\end{array}\right]+\left[\begin{array}{c}
L_{A} \\
0
\end{array}\right] V\left[\begin{array}{ll}
0 & R_{B} P
\end{array}\right] } \\
& +\left(\left[\begin{array}{c}
L_{A} \\
0
\end{array}\right] V\left[\begin{array}{ll}
0 & R_{B} P
\end{array}\right]\right)^{*} .
\end{aligned}
$$

Applying Lemma 1 to (19) and (20) yields

$$
\max _{V} r[q(V)]=\min \left\{r(M), r\left(M_{1}\right), r\left(M_{2}\right)\right\},
$$

$\min _{V} r[q(V)]$

$$
\begin{gathered}
=2 r(M)+\max \left\{s_{+}+s_{-}, t_{+}+t_{-}, s_{+}+t_{-}, s_{-}+t_{+}\right\}, \\
\max _{V} i_{ \pm}[q(V)]=\min \left\{i_{ \pm}\left(M_{1}\right), i_{ \pm}\left(M_{2}\right)\right\}, \\
\min _{V} i_{ \pm}[q(V)]=r(M)+\max \left\{s_{ \pm}, t_{ \pm}\right\},
\end{gathered}
$$

where

$$
\begin{gathered}
M=\left[\begin{array}{cccc}
Q & X_{0} P & L_{A} & 0 \\
P X_{0}^{*} & P & 0 & P R_{B}
\end{array}\right], \quad M_{1}=\left[\begin{array}{ccc}
Q & X_{0} P & L_{A} \\
P X_{0}^{*} & P & 0 \\
L_{A} & 0 & 0
\end{array}\right], \\
M_{2}=\left[\begin{array}{ccc}
Q & X_{0} P & 0 \\
P X_{0}^{*} & P & P R_{B} \\
0 & R_{B} P & 0
\end{array}\right], \quad M_{3}=\left[\begin{array}{cccc}
Q & X_{0} P & L_{A} & 0 \\
P X_{0}^{*} & P & 0 & P R_{B} \\
L_{A} & 0 & 0 & 0
\end{array}\right], \\
M_{4}=\left[\begin{array}{cccc}
Q & X_{0} P & L_{A} & 0 \\
P X_{0}^{*} & P & 0 & P R_{B} \\
0 & R_{B} P & 0 & 0
\end{array}\right], \\
s_{ \pm}=i_{ \pm}\left(M_{1}\right)-r\left(M_{3}\right), \quad t_{ \pm}=i_{ \pm}\left(M_{2}\right)-r\left(M_{4}\right) .
\end{gathered}
$$


Applying Lemmas 2 and 3, elementary matrix operations and congruence matrix operations, we obtain

$$
\begin{gathered}
r(M)=n+r\left[\begin{array}{ccc}
0 & P & P \\
A Q & C P & 0 \\
-D^{*} & 0 & B^{*}
\end{array}\right]-r(A)-r(B), \\
r\left(M_{1}\right)=2 n+r\left(A Q A^{*}-C P C^{*}\right)-2 r(A)+r(P), \\
i_{ \pm}\left(M_{1}\right)=n+i_{ \pm}\left(A Q A^{*}-C P C^{*}\right)-r(A)+i_{ \pm}(P), \\
r\left(M_{2}\right)=r\left[\begin{array}{ccc}
Q & 0 & D \\
0 & -P & B \\
D^{*} & B^{*} & 0
\end{array}\right]-2 r(B)+r(P), \\
i_{ \pm}\left(M_{2}\right)=i_{ \pm}\left[\begin{array}{ccc}
Q & 0 & D \\
0 & -P & B \\
D^{*} & B^{*} & 0
\end{array}\right]-r(B)+i_{ \pm}(P), \\
r\left(M_{3}\right)=2 n+r(P)-2 r(A)-r(B)+r\left[\begin{array}{ll}
C P & A Q A^{*} \\
B^{*} & D^{*} A^{*}
\end{array}\right], \\
r\left(M_{4}\right)=n+r(P)+r\left[\begin{array}{ccc}
0 & P & B \\
A Q & C P & 0 \\
D^{*} & B^{*} & 0
\end{array}\right]-2 r(B)-r(A) .
\end{gathered}
$$

Substituting (24) into (22), we obtain the results.

Using immediately Theorem 5, we can easily get the following.

Theorem 6. Let $f(X)$ be as given in (4), $s_{ \pm}$and let $t_{ \pm}$be as given in Theorem 5 and assume that $A X=C$ and $X B=D$ in (5) are consistent. Then we have the following.

(a) $A X=C$ and $X B=D$ have a common solution such that $Q-X P X^{*} \geq 0$ if and only if

$$
\begin{gathered}
n+r\left[\begin{array}{ccc}
0 & P & P \\
A Q & C P & 0 \\
-D^{*} & 0 & B^{*}
\end{array}\right]-r(A)-r(B)-i_{-}(P)+s_{-} \leq 0, \\
n+r\left[\begin{array}{ccc}
0 & P & P \\
A Q & C P & 0 \\
-D^{*} & 0 & B^{*}
\end{array}\right]-r(A)-r(B)-i_{-}(P)+t_{-} \leq 0 .
\end{gathered}
$$

(b) $A X=C$ and $X B=D$ have a common solution such that $Q-X P X^{*} \leq 0$ if and only if

$$
\begin{gathered}
n+r\left[\begin{array}{ccc}
0 & P & P \\
A Q & C P & 0 \\
-D^{*} & 0 & B^{*}
\end{array}\right]-r(A)-r(B)-i_{+}(P)+s_{+} \leq 0, \\
n+r\left[\begin{array}{ccc}
0 & P & P \\
A Q & C P & 0 \\
-D^{*} & 0 & B^{*}
\end{array}\right]-r(A)-r(B)-i_{+}(P)+t_{+} \leq 0 .
\end{gathered}
$$

(c) $A X=C$ and $X B=D$ have a common solution such that $Q-X P X^{*}>0$ if and only if

$$
\begin{gathered}
i_{+}\left(A Q A^{*}-C P C^{*}\right)-r(A) \geq 0, \\
i_{+}\left[\begin{array}{ccc}
Q & 0 & D \\
0 & -P & B \\
D^{*} & B^{*} & 0
\end{array}\right]-r(B) \geq n .
\end{gathered}
$$

(d) $A X=C$ and $X B=D$ have a common solution such that $Q-X P X^{*}<0$ if and only if

$$
\begin{gathered}
i_{-}\left(A Q A^{*}-C P C^{*}\right)-r(A) \geq 0, \\
i_{-}\left[\begin{array}{ccc}
Q & 0 & D \\
0 & -P & B \\
D^{*} & B^{*} & 0
\end{array}\right]-r(B) \geq n .
\end{gathered}
$$

(e) All common solutions of $A X=C$ and $X B=D$ satisfy $Q-X P X^{*} \geq 0$ if and only if

$$
\begin{gathered}
n+i_{-}\left(A Q A^{*}-C P C^{*}\right)-r(A)=0, \\
\text { or, } i_{-}\left[\begin{array}{ccc}
Q & 0 & D \\
0 & -P & B \\
D^{*} & B^{*} & 0
\end{array}\right]-r(B)=0 .
\end{gathered}
$$

(f) All common solutions of $A X=C$ and $X B=D$ satisfy $Q-X P X^{*} \leq 0$ if and only if

$$
\begin{gathered}
n+i_{+}\left(A Q A^{*}-C P C^{*}\right)-r(A)=0, \\
\text { or, } i_{+}\left[\begin{array}{ccc}
Q & 0 & D \\
0 & -P & B \\
D^{*} & B^{*} & 0
\end{array}\right]-r(B)=0 .
\end{gathered}
$$

(g) All common solutions of $A X=C$ and $X B=D$ satisfy $Q-X P X^{*}>0$ if and only if

$$
n+r\left[\begin{array}{ccc}
0 & P & P \\
A Q & C P & 0 \\
-D^{*} & 0 & B^{*}
\end{array}\right]-r(A)-r(B)-i_{+}(P)+s_{+}=n,
$$

$$
n+r\left[\begin{array}{ccc}
0 & P & P \\
A Q & C P & 0 \\
-D^{*} & 0 & B^{*}
\end{array}\right]-r(A)-r(B)-i_{+}(P)+t_{+}=n \text {. }
$$

(h) All common solutions of $A X=C$ and $X B=D$ satisfy $Q-X P X^{*}<0$ if and only if

$$
n+r\left[\begin{array}{ccc}
0 & P & P \\
A Q & C P & 0 \\
-D^{*} & 0 & B^{*}
\end{array}\right]-r(A)-r(B)-i_{-}(P)+s_{-}=n \text {, }
$$

$$
n+r\left[\begin{array}{ccc}
0 & P & P \\
A Q & C P & 0 \\
-D^{*} & 0 & B^{*}
\end{array}\right]-r(A)-r(B)-i_{-}(P)+t_{-}=n \text {. }
$$


(i) $A X=C, X B=D$, and $Q=X P X^{*}$ have a common solution if and only if

$2 n+2 r\left[\begin{array}{ccc}0 & P & P \\ A Q & C P & 0 \\ -D^{*} & 0 & B^{*}\end{array}\right]-2 r(A)-2 r(B)-r(P)+s_{+}+s_{-} \leq 0$,

$2 n+2 r\left[\begin{array}{ccc}0 & P & P \\ A Q & C P & 0 \\ -D^{*} & 0 & B^{*}\end{array}\right]-2 r(A)-2 r(B)-r(P)+t_{+}+t_{-} \leq 0$,

$2 n+2 r\left[\begin{array}{ccc}0 & P & P \\ A Q & C P & 0 \\ -D^{*} & 0 & B^{*}\end{array}\right]-2 r(A)-2 r(B)-r(P)+s_{+}+t_{-} \leq 0$,

$2 n+2 r\left[\begin{array}{ccc}0 & P & P \\ A Q & C P & 0 \\ -D^{*} & 0 & B^{*}\end{array}\right]-2 r(A)-2 r(B)-r(P)+s_{-}+t_{+} \leq 0$.

Let $P=I$ in Theorem 5, we get the following corollary.

Corollary 7. Let $Q \in \mathbb{C}^{n \times n}, A, B, C$, and $D$ be given. Assume that (5) is consistent. Denote

$$
\begin{gathered}
T_{1}=\left[\begin{array}{cc}
C & A Q \\
B^{*} & D^{*}
\end{array}\right], \quad T_{2}=A Q A^{*}-C C^{*}, \\
T_{3}=\left[\begin{array}{cc}
Q & D \\
D^{*} & B^{*} B
\end{array}\right], \quad T_{4}=\left[\begin{array}{cc}
C & A Q A^{*} \\
B^{*} & D^{*} A^{*}
\end{array}\right], \\
T_{5}=\left[\begin{array}{cc}
C B & A Q \\
B^{*} B & D^{*}
\end{array}\right] .
\end{gathered}
$$

Then,

$$
\begin{gathered}
\max _{A X=C, X B=D} r\left(Q-X X^{*}\right) \\
=\min \left\{n+r\left(T_{1}\right)-r(A)-r(B), 2 n+r\left(T_{2}\right)\right. \\
\left.-2 r(A), n+r\left(T_{3}\right)-2 r(B)\right\}, \\
\min _{A X=C, X B=D} r\left(Q-X X^{*}\right) \\
=2 r\left(T_{1}\right)+\max \left\{r\left(T_{2}\right)-2 r\left(T_{4}\right),-n+r\left(T_{3}\right)\right. \\
-2 r\left(T_{5}\right), i_{+}\left(T_{2}\right)+i_{-}\left(T_{3}\right) \\
-r\left(T_{4}\right)-r\left(T_{5}\right),-n+i_{-}\left(T_{2}\right) \\
\left.+i_{+}\left(T_{3}\right)-r\left(T_{4}\right)-r\left(T_{5}\right)\right\} \\
\max _{A X=C, X B=D} i_{+}\left(Q-X X^{*}\right) \\
=\min \left\{n+i_{+}\left(T_{2}\right)-r(A), i_{+}\left(T_{3}\right)-r(B)\right\},
\end{gathered}
$$

$$
\begin{aligned}
& \max _{A X=C, X B=D} i_{-}\left(Q-X X^{*}\right) \\
& =\min \left\{n+i_{-}\left(T_{2}\right)-r(A), n+i_{-}\left(T_{3}\right)-r(B)\right\}, \\
& \min _{A X=C, X B=D} i_{+}\left(Q-X X^{*}\right) \\
& =r\left(T_{1}\right)+\max \left\{i_{+}\left(T_{2}\right)-r\left(T_{4}\right), i_{+}\left(T_{3}\right)-n-r\left(T_{5}\right)\right\}, \\
& \min _{A X=C, X B=D} i_{-}\left(Q-X X^{*}\right) \\
& =r\left(T_{1}\right)+\max \left\{i_{-}\left(T_{2}\right)-r\left(T_{4}\right), i_{-}\left(T_{3}\right)-r\left(T_{5}\right)\right\} .
\end{aligned}
$$

Remark 8. Corollary 7 is one of the results in [24].

Let $B$ and $D$ vanish in Theorem 5 , then we can obtain the maximal and minimal ranks and inertias of (4) subject to $A X=C$.

Corollary 9. Let $f(X)$ be as given in (4) and assume that $A X=C$ is consistent. Then

$$
\begin{aligned}
\max _{A X=C} r & \left(Q-X P X^{*}\right) \\
= & \min \{n+r[A Q C P]-r(A)-r(B), \\
& \left.2 n+r\left(A Q A^{*}-C P C^{*}\right)-2 r(A), r(Q)+r(P)\right\} \\
\min _{A X=C} r\left(Q-X P X^{*}\right) & \\
= & 2 n+2 r[A Q C P]-2 r(A) \\
& +\max \left\{s_{+}+s_{-}, t_{+}+t_{-}, s_{+}+t_{-}, s_{-}+t_{+}\right\}, \\
\max _{A X=C} i_{ \pm} & \left(Q-X P X^{*}\right) \\
= & \min \left\{n+i_{ \pm}\left(A Q A^{*}-C P C^{*}\right)-r(A), i_{ \pm}(Q)+i_{\mp}(P)\right\}, \\
\min _{A X=C} i_{ \pm} & \left(Q-X P X^{*}\right) \\
= & n+r[A Q C P]-r(A)+i_{\mp}(P)+\max \left\{s_{ \pm}, t_{ \pm}\right\},
\end{aligned}
$$

where

$$
\begin{aligned}
s_{ \pm}= & -n+r(A)-i_{\mp}(P) \\
& +i_{ \pm}\left(A Q A^{*}-C P C^{*}\right)-r\left[C P \quad A Q A^{*}\right], \\
t_{ \pm}= & -n+r(A)+i_{ \pm}(Q)-r(P)-\left[\begin{array}{ll}
A Q & C P
\end{array}\right] .
\end{aligned}
$$

Remark 10. Corollary 9 is one of the results in [22].

\section{References}

[1] D. L. Chu, Y. S. Hung, and H. J. Woerdeman, "Inertia and rank characterizations of some matrix expressions," SIAM Journal on Matrix Analysis and Applications, vol. 31, no. 3, pp. 1187-1226, 2009.

[2] Z. H. He and Q. W. Wang, "Solutions to optimization problems on ranks and inertias of a matrix function with applications," 
Applied Mathematics and Computation, vol. 219, no. 6, pp. 29893001, 2012.

[3] Y. Liu and Y. Tian, "Max-min problems on the ranks and inertias of the matrix expressions $A-B X C \pm(B X C)^{*}$ with applications," Journal of Optimization Theory and Applications, vol. 148, no. 3, pp. 593-622, 2011.

[4] G. Marsaglia and G. P. H. Styan, "Equalities and inequalities for ranks of matrices," Linear and Multilinear Algebra, vol. 2, pp. 269-292, 1974/75.

[5] X. Zhang, Q. W. Wang, and X. Liu, "Inertias and ranks of some Hermitian matrix functions with applications," Central European Journal of Mathematics, vol. 10, no. 1, pp. 329-351, 2012.

[6] Q. W. Wang, Y. Zhou, and Q. Zhang, "Ranks of the common solution to six quaternion matrix equations," Acta Mathematicae Applicatae Sinica, vol. 27, no. 3, pp. 443-462, 2011.

[7] Q. Zhang and Q. W. Wang, “The $(P, Q)$-(skew)symmetric extremal rank solutions to a system of quaternion matrix equations," Applied Mathematics and Computation, vol. 217, no. 22, pp. 9286-9296, 2011.

[8] D. Z. Lian, Q. W. Wang, and Y. Tang, "Extreme ranks of a partial banded block quaternion matrix expression subject to some matrix equations with applications," Algebra Colloquium, vol. 18, no. 2, pp. 333-346, 2011.

[9] H. S. Zhang and Q. W. Wang, "Ranks of submatrices in a general solution to a quaternion system with applications," Bulletin of the Korean Mathematical Society, vol. 48, no. 5, pp. 969-990, 2011.

[10] Q. W. Wang and J. Jiang, "Extreme ranks of (skew-)Hermitian solutions to a quaternion matrix equation," Electronic Journal of Linear Algebra, vol. 20, pp. 552-573, 2010.

[11] I. A. Khan, Q. W. Wang, and G. J. Song, "Minimal ranks of some quaternion matrix expressions with applications," Applied Mathematics and Computation, vol. 217, no. 5, pp. 2031-2040, 2010.

[12] Q. Wang, S. Yu, and W. Xie, "Extreme ranks of real matrices in solution of the quaternion matrix equation $A X B=C$ with applications," Algebra Colloquium, vol. 17, no. 2, pp. 345-360, 2010.

[13] Q. W. Wang, H. S. Zhang, and G. J. Song, "A new solvable condition for a pair of generalized Sylvester equations," Electronic Journal of Linear Algebra, vol. 18, pp. 289-301, 2009.

[14] Q. W. Wang, S. W. Yu, and Q. Zhang, "The real solutions to a system of quaternion matrix equations with applications," Communications in Algebra, vol. 37, no. 6, pp. 2060-2079, 2009.

[15] Q. W. Wang and C. K. Li, "Ranks and the least-norm of the general solution to a system of quaternion matrix equations," Linear Algebra and its Applications, vol. 430, no. 5-6, pp. 1626$1640,2009$.

[16] Q. W. Wang, H. S. Zhang, and S. W. Yu, "On solutions to the quaternion matrix equation $A X B+C Y D=$ E, Electronic Journal of Linear Algebra, vol. 17, pp. 343-358, 2008.

[17] Q. W. Wang, G. J. Song, and X. Liu, "Maximal and minimal ranks of the common solution of some linear matrix equations over an arbitrary division ring with applications," Algebra Colloquium, vol. 16, no. 2, pp. 293-308, 2009.

[18] Q. W. Wang, G. J. Song, and C. Y. Lin, "Rank equalities related to the generalized inverse $A_{T, S}^{(2)}$ with applications," Applied Mathematics and Computation, vol. 205, no. 1, pp. 370-382, 2008.
[19] Q. W. Wang, S. W. Yu, and C. Y. Lin, "Extreme ranks of a linear quaternion matrix expression subject to triple quaternion matrix equations with applications," Applied Mathematics and Computation, vol. 195, no. 2, pp. 733-744, 2008.

[20] Q. W. Wang, G. J. Song, and C. Y. Lin, "Extreme ranks of the solution to a consistent system of linear quaternion matrix equations with an application," Applied Mathematics and Computation, vol. 189, no. 2, pp. 1517-1532, 2007.

[21] Y. Tian, "Formulas for calculating the extremum ranks and inertias of a four-term quadratic matrix-valued function and their applications," Linear Algebra and its Applications, vol. 437, no. 3, pp. 835-859, 2012.

[22] Y. Tian, "Solving optimization problems on ranks and inertias of some constrained nonlinear matrix functions via an algebraic linearization method," Nonlinear Analysis. Theory, Methods \& Applications, vol. 75, no. 2, pp. 717-734, 2012.

[23] Y. Tian, "Maximization and minimization of the rank and inertia of the Hermitian matrix expression $A-B X-(B X)^{*}$ with applications," Linear Algebra and its Applications, vol. 434, no. 10, pp. 2109-2139, 2011.

[24] Q. W. Wang, X. Zhang, and Z. H. He, "On the Hermitian structures of the solution to a pair of matrix equations," Linear and Multilinear Algebra, vol. 61, no. 1, pp. 73-90, 2013. 


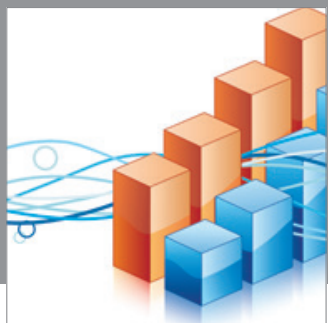

Advances in

Operations Research

mansans

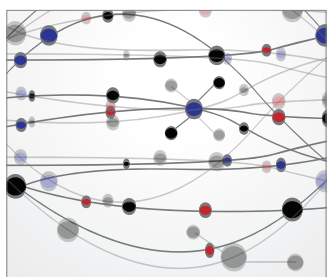

The Scientific World Journal
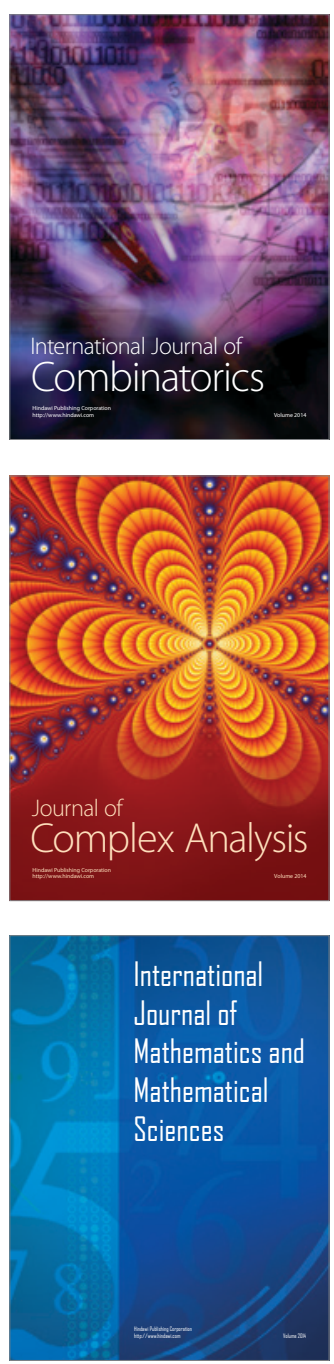
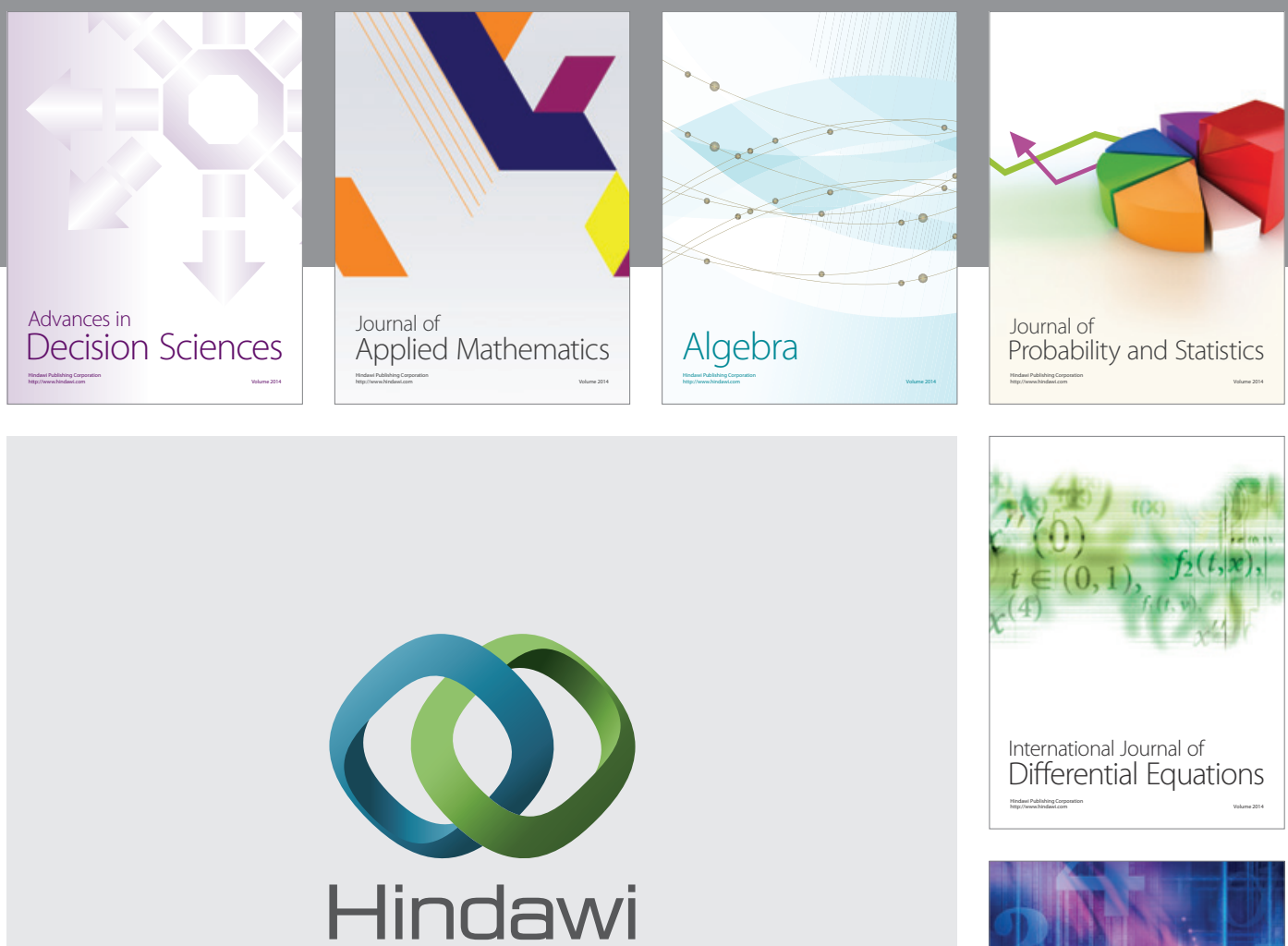

Submit your manuscripts at http://www.hindawi.com
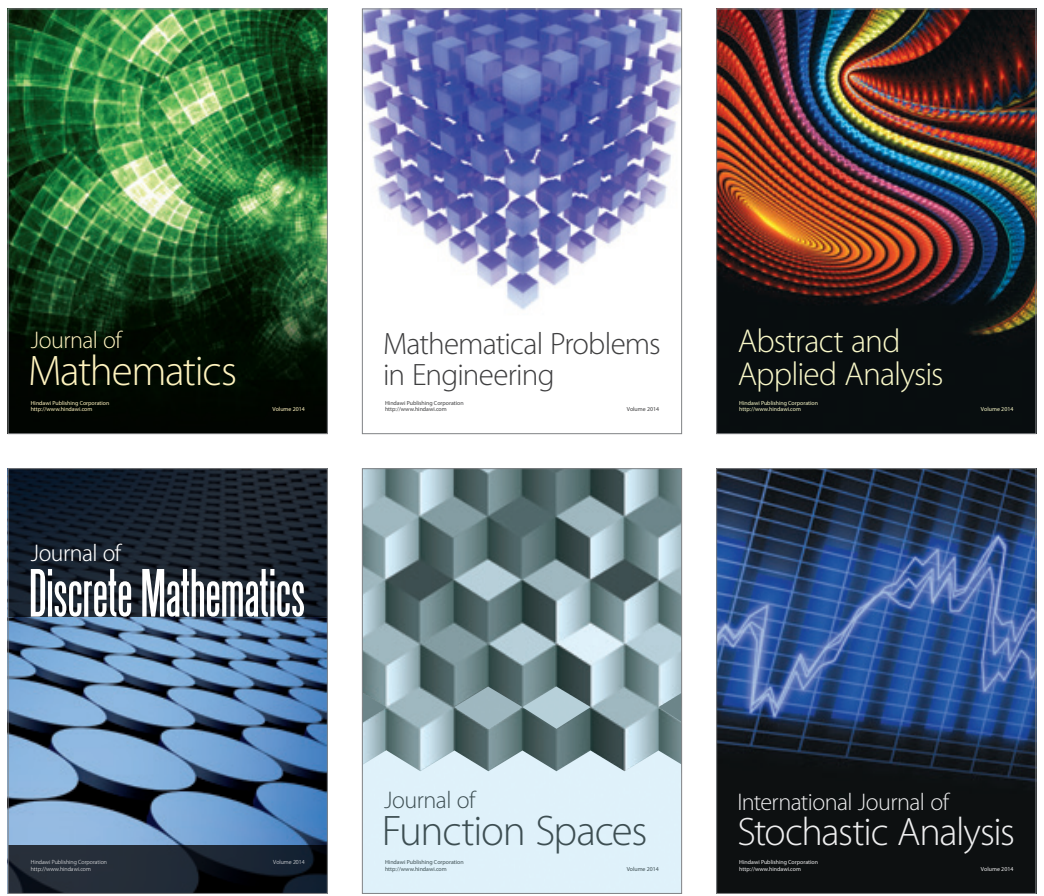

Journal of

Function Spaces

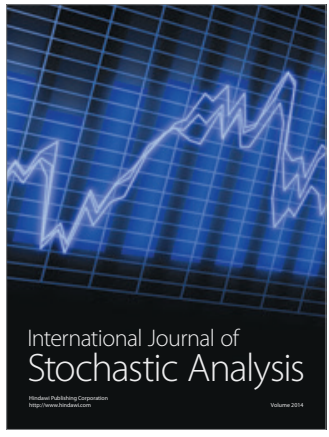

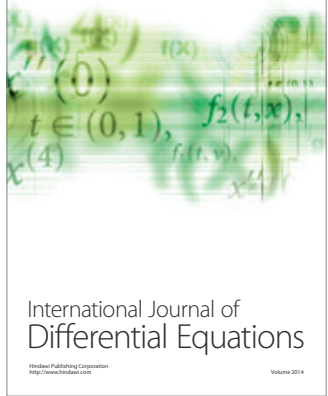
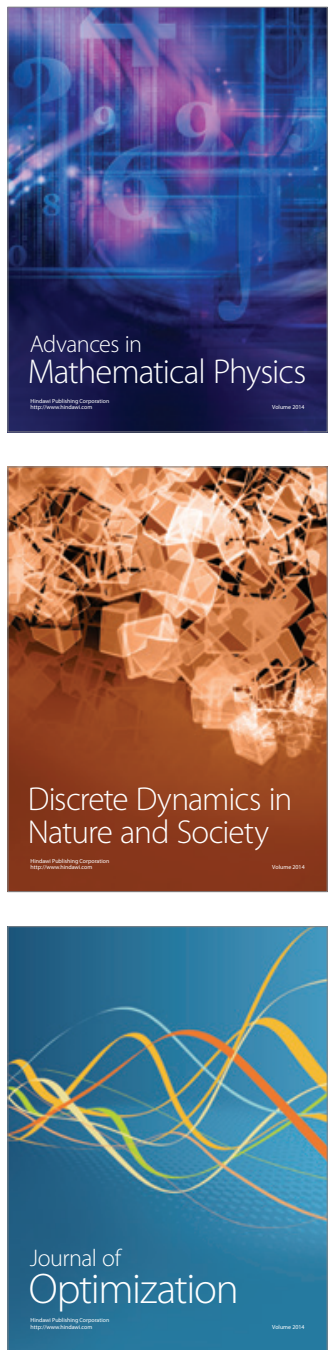